\title{
Turning the screw: engineering extreme pH resistance in Escherichia coli through combinatorial synthetic operons
}

by

Guilherme M. V. de Siqueira $^{1}$, Rafael Silva-Rocha ${ }^{2}$ and María-Eugenia Guazzaroni ${ }^{3 *}$

${ }^{1}$ Departamento de Bioquímica, Faculdade de Medicina de Ribeirão Preto (FMRP-USP), Ribeirão Preto, SP, Brasil. 14049-900

${ }^{2}$ Departamento de Biologia Celular e Molecular e Bioagentes Patogênicos, Faculdade de Medicina de Ribeirão Preto (FMRP-USP), Ribeirão Preto, SP, Brasil. 14049-900

${ }^{3}$ Departamento de Biologia, Faculdade de Filosofia Ciências e Letras de Ribeirão Preto (FFCLRP-USP), Ribeirão Preto, SP, Brasil. 14040-901

\section{Supporting Information}

Figure S1. Characterization of the RBSs used in this study 2

Figure S2. Growth profiles of clones harboring pARC211 and the pSEVA232 empty vector.... 3

Figure S3. Survival profiles for different pARC strains after $1 \mathrm{~h}$ of acidic shock. ...................... 4

Figure S4. Relationship analysis between acid resistance after $1 \mathrm{~h}$ and the fitness cost of different clones harboring plasmids with combinations of the three RBS .............................................. 5

Figure S5. Measured growth rates for determination of the fitness costs .................................. 6

Table S1. Survival percentages obtained at time points $1 \mathrm{~h}$ and $2 \mathrm{~h}$ of the acidic challenge. ...... 7

Table S2. Growth rate and fitness cost of strains grown in minimal medium............................ 8

Table S3. Oligonucleotides used for assembly of synthetic operons. ....................................... 9 


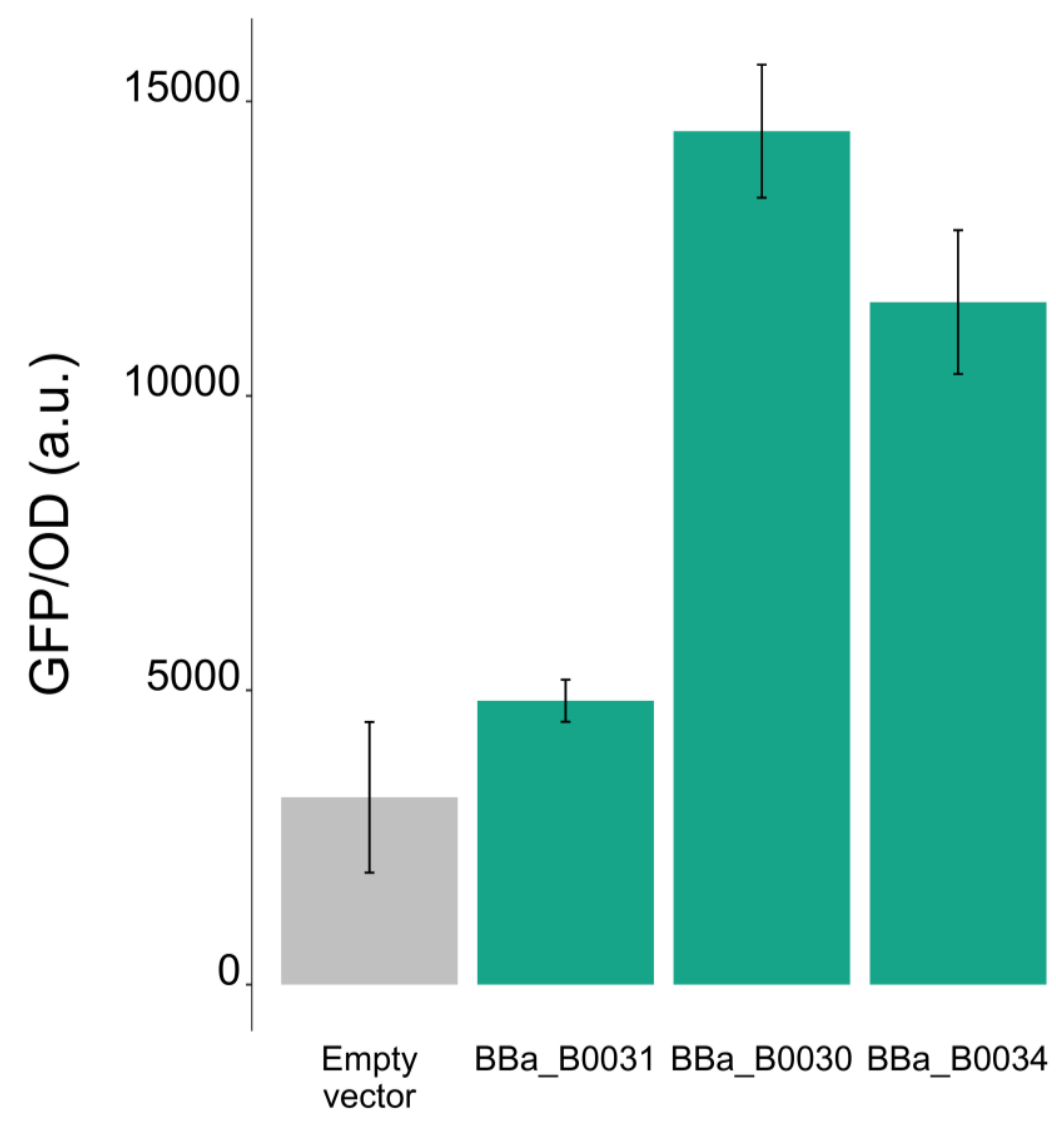

Figure S1. Characterization of the RBSs used in this study. As measured by GFPlva fluorescence, considering RBS3 (Bba_B0034) as the standard for 100\% translational strength, we calculate relative strengths of $125 \%$ for RBS2 (Bba_B0030) and 41,6\% for RBS1 (Bba_B0031), which are different values from the ones previously reported in the iGEM database (60\% and 7\%, respectively). RBS efficiency is heavily dependent on the broader context of mRNA secondary structure, thus the difference between the reported values and the ones obtained in this study may be due to the different genetic contexts in which these RBS are inserted and the conditions in which they were characterized, revealing a non-orthogonal behavior of these genetic parts. a.u.= arbitrary units. 

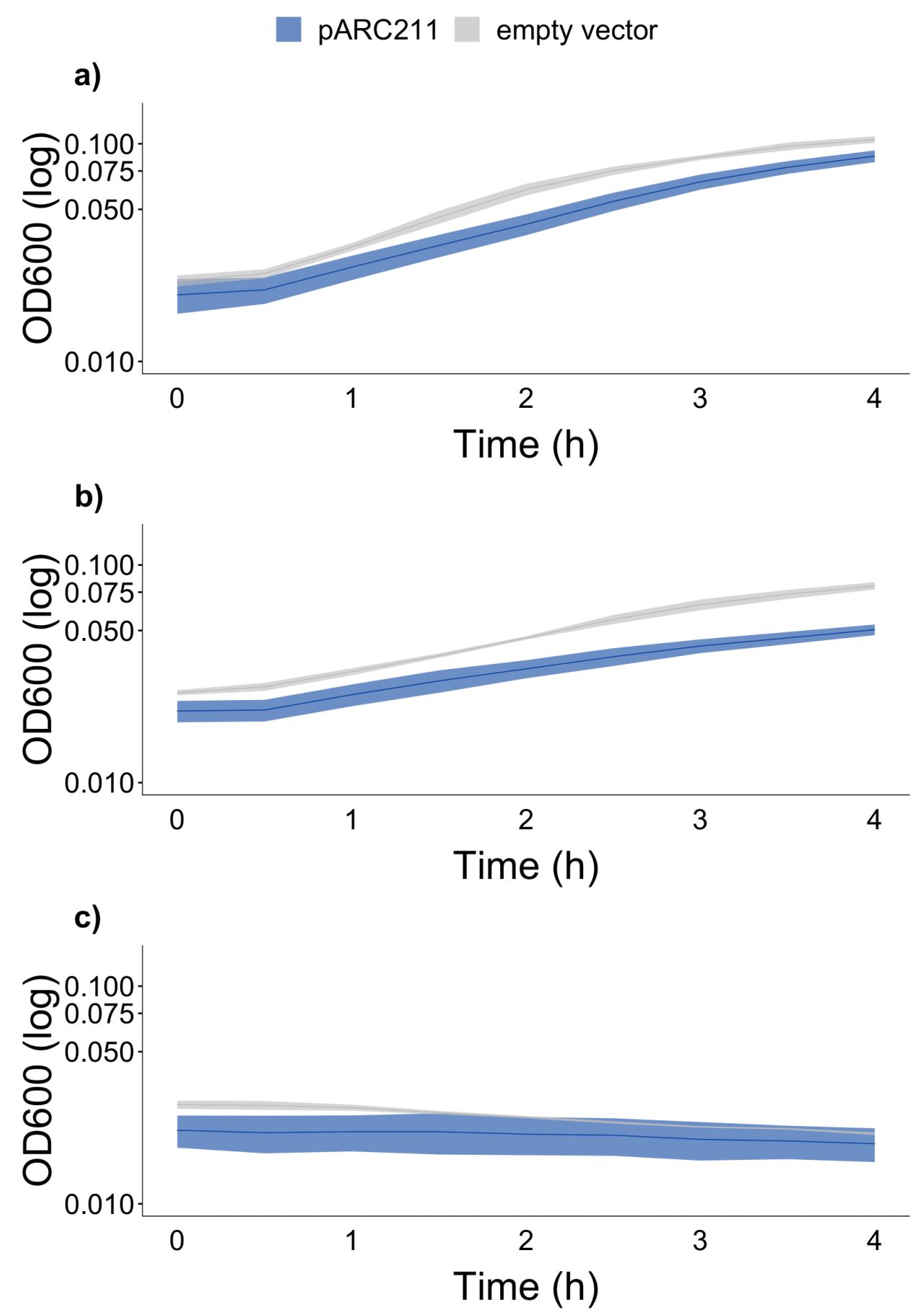

Figure S2. Growth profiles of clones harboring pARC211 and the pSEVA232 empty vector at a) neutral $\mathrm{pH}$; b) $\mathrm{pH} 3.5$; c) $\mathrm{pH} 3$. 


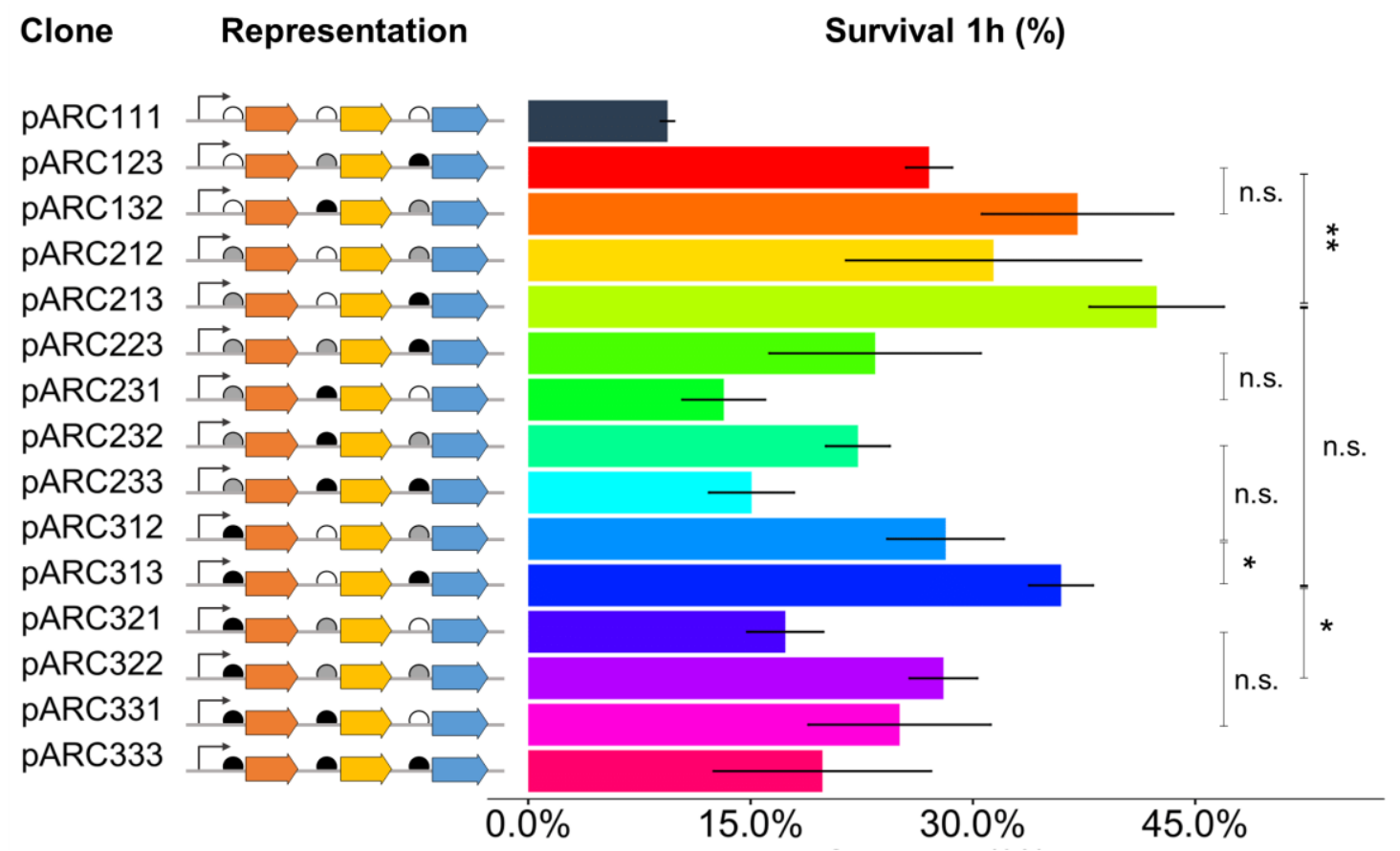

Figure S3. Extended survival profiles for different pARC strains after $1 \mathrm{~h}$ of acidic shock. Error bars indicate standard deviation from three independent experiments and statistical significance is represented as asterisks, considering $p$ values as follows: $\mathrm{ns}, p>0.05$; $p$ $\leq 0.05 ; * * p \leq 0.01 ; * * * p \leq 0.001 ; * * * * p \leq 0.0001$. 


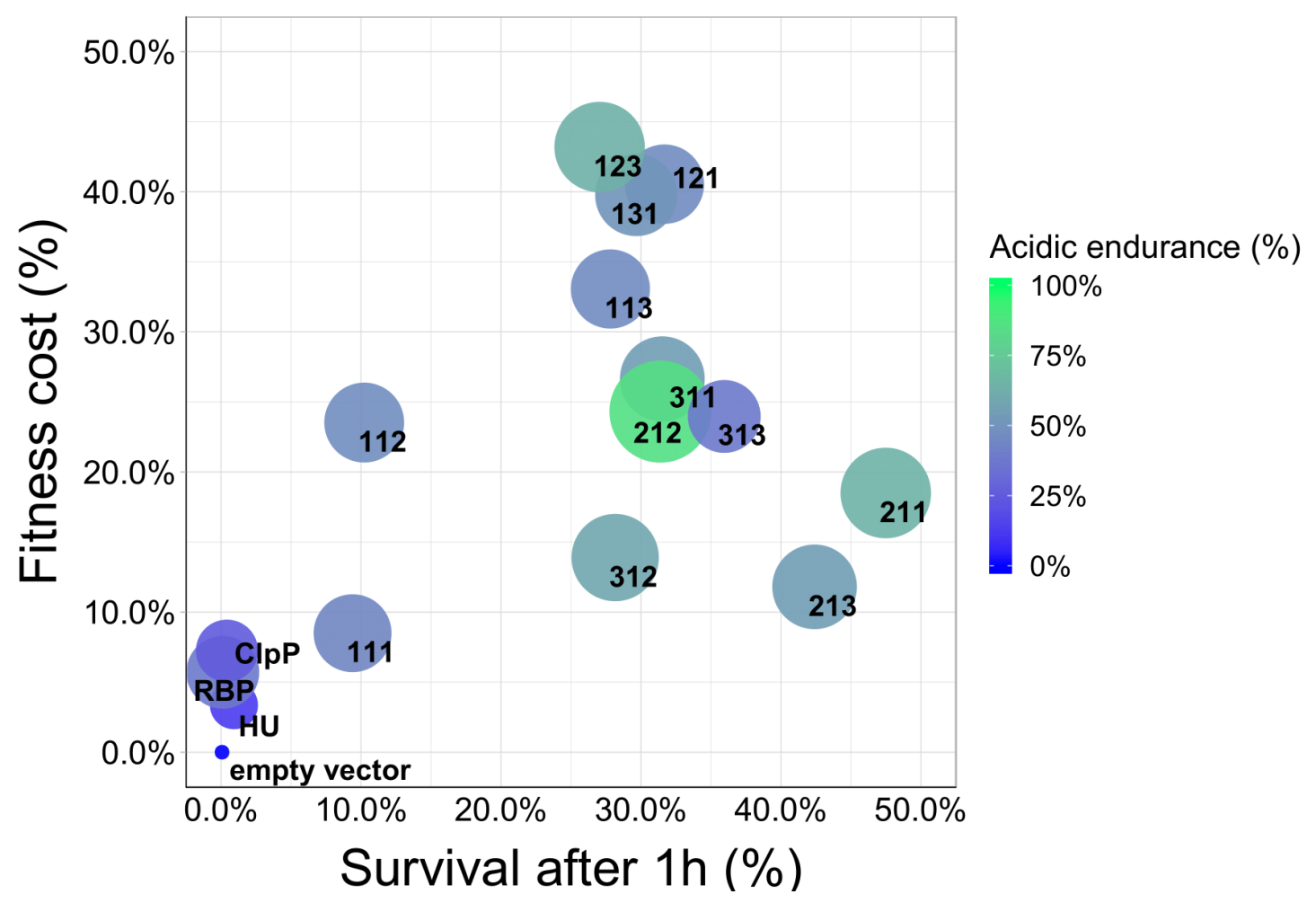

Figure S4. Relationship analysis between acid resistance after $1 \mathrm{~h}$ and the fitness cost of different clones harboring plasmids with combinations of the three RBS. The size and color of the circles vary in relation to the acidic endurance of the depicted strains. 


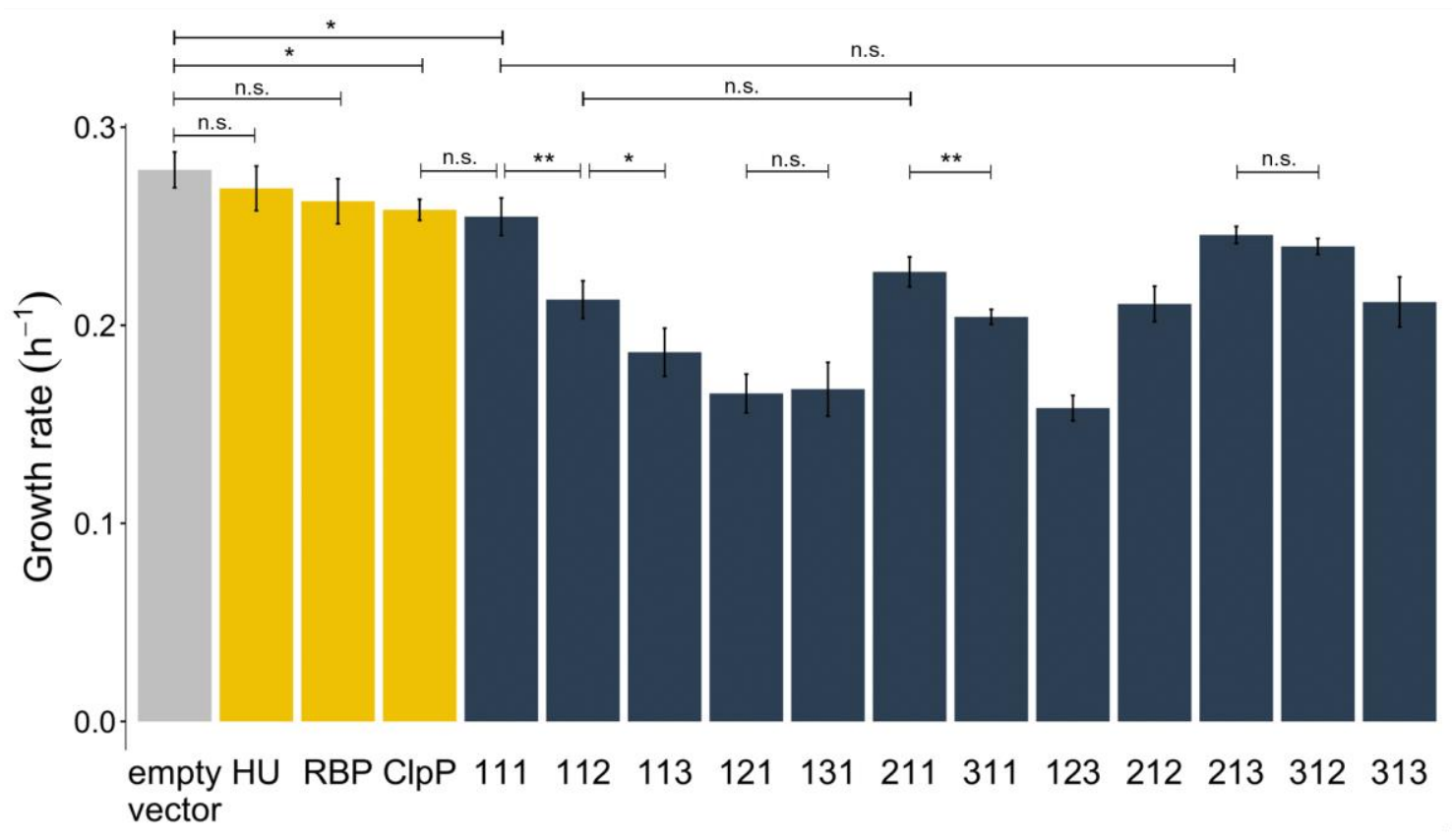

Figure S5. Measured growth rates for determination of the fitness costs. Error bars indicate standard deviation from three independent experiments, and statistical significance is represented as asterisks, considering $p$ values as follows: ns, $p>0.05 ;{ }^{*} p$ $\leq 0.05 ; * * p \leq 0.01 ; * * * p \leq 0.001 ; * * * * p \leq 0.0001$. 
Table S1. Survival percentages obtained at time points $1 \mathrm{~h}$ and $2 \mathrm{~h}$ of the acidic challenge. $\mathrm{SP}=$ Survival Percentage, $\mathrm{sd}=$ standard deviation from three independent experiments.

\begin{tabular}{|c|c|c|c|c|}
\hline Strain & SP (1h) & sd (1h) & SP (2h) & sd (2h) \\
\hline pSEVA232 & $0.071 \%$ & $0.008 \%$ & $0.000 \%$ & $0.000 \%$ \\
\hline pARC300 (HU) & $0.921 \%$ & $0.089 \%$ & $0.115 \%$ & $0.046 \%$ \\
\hline pARC030 (RBP) & $0.124 \%$ & $0.026 \%$ & $0.047 \%$ & $0.077 \%$ \\
\hline pARC003 (ClpP) & $0.422 \%$ & $0.115 \%$ & $0.107 \%$ & $0.084 \%$ \\
\hline pARC111 & $9.396 \%$ & $0.452 \%$ & $4.205 \%$ & $0.474 \%$ \\
\hline pARC112 & $10.227 \%$ & $0.249 \%$ & $4.861 \%$ & $0.141 \%$ \\
\hline pARC113 & $27.814 \%$ & $0.789 \%$ & $12.938 \%$ & $2.610 \%$ \\
\hline pARC121 & $31.673 \%$ & $3.697 \%$ & $14.820 \%$ & $0.670 \%$ \\
\hline pARC131 & $29.658 \%$ & $3.766 \%$ & $15.107 \%$ & $1.729 \%$ \\
\hline pARC211 & $47.484 \%$ & $3.289 \%$ & $30.576 \%$ & $5.485 \%$ \\
\hline pARC311 & $31.522 \%$ & $3.880 \%$ & $17.361 \%$ & $1.440 \%$ \\
\hline pARC123 & $27.045 \%$ & $1.566 \%$ & $17.374 \%$ & $0.708 \%$ \\
\hline pARC212 & $31.389 \%$ & $9.953 \%$ & $26.797 \%$ & $6.601 \%$ \\
\hline pARC213 & $42.399 \%$ & $4.540 \%$ & $23.205 \%$ & $8.493 \%$ \\
\hline pARC312 & $28.156 \%$ & $3.936 \%$ & $16.624 \%$ & $4.870 \%$ \\
\hline pARC313 & $35.948 \%$ & $2.158 \%$ & $13.614 \%$ & $1.291 \%$ \\
\hline pARC132 & $37.062 \%$ & $6.462 \%$ & $16.961 \%$ & $3.478 \%$ \\
\hline pARC223 & $23.401 \%$ & $7.135 \%$ & $16.606 \%$ & $6.769 \%$ \\
\hline pARC231 & $13.190 \%$ & $2.794 \%$ & $3.078 \%$ & $1.553 \%$ \\
\hline pARC232 & $22.240 \%$ & $2.158 \%$ & $17.039 \%$ & $2.405 \%$ \\
\hline pARC233 & $15.065 \%$ & $2.868 \%$ & $7.990 \%$ & $1.840 \%$ \\
\hline pARC321 & $17.340 \%$ & $2.571 \%$ & $14.350 \%$ & $1.811 \%$ \\
\hline pARC322 & $28.011 \%$ & $2.290 \%$ & $16.186 \%$ & $1.087 \%$ \\
\hline pARC331 & $25.050 \%$ & $6.151 \%$ & $20.652 \%$ & $5.243 \%$ \\
\hline pARC333 & $19.846 \%$ & $7.338 \%$ & $14.296 \%$ & $6.582 \%$ \\
\hline
\end{tabular}


Table S2. Growth rate and fitness cost of strains grown in minimal medium. As stated in the text. growth rates were measured on the linear phase of growth under $\mathrm{pH} 7$, and fitness cost was calculated by the comparison of such rates with the growth rate of the strain used as negative control, which lacks the expression of heterologous genes. $s d=$ standard deviation from three independent experiments.

\begin{tabular}{|c|c|c|c|}
\hline Strain & Growth rate $\left(h^{-1}\right)$ & sd & Fitness cost $(\%)$ \\
\hline pSEVA232 & 0.2785 & 0.0090 & $0.00 \%$ \\
\hline pARC300 (HU) & 0.2691 & 0.0113 & $3.36 \%$ \\
\hline pARC030 (RBP) & 0.2626 & 0.0114 & $5.70 \%$ \\
\hline pARC003 (ClpP) & 0.2583 & 0.0053 & $7.24 \%$ \\
\hline pARC111 & 0.2548 & 0.0095 & $8.49 \%$ \\
\hline pARC112 & 0.2130 & 0.0095 & $23.53 \%$ \\
\hline pARC113 & 0.1864 & 0.0121 & $33.06 \%$ \\
\hline pARC121 & 0.1656 & 0.0098 & $40.54 \%$ \\
\hline pARC131 & 0.1677 & 0.0136 & $39.77 \%$ \\
\hline pARC211 & 0.2270 & 0.0075 & $18.50 \%$ \\
\hline pARC311 & 0.2043 & 0.0038 & $26.65 \%$ \\
\hline pARC123 & 0.1582 & 0.0064 & $43.19 \%$ \\
\hline pARC212 & 0.2108 & 0.0089 & $24.31 \%$ \\
\hline pARC213 & 0.2456 & 0.0043 & $11.80 \%$ \\
\hline pARC312 & 0.2398 & 0.0040 & $13.89 \%$ \\
\hline pARC313 & 0.2117 & 0.0126 & $23.96 \%$ \\
\hline
\end{tabular}


Table S3. Oligonucleotides used for assembly of synthetic operons. RBS sequences are shown in bold and the annealing sequence to the target gene are underlined. R.S. = Restriction Site

\begin{tabular}{|c|c|c|}
\hline ID & Sequence $(5, \rightarrow$ 3') & R.S. \\
\hline HUF60 & CGCGAAGCTTATTAAAGAGGAGAAATACTAGATGAGCAAAGAAAAAGAAGTGCT & HindIII \\
\hline HUF7 & CGCGAAGCTTTCACACAGGAAACCTACTAGATGAGCAAAGAAAAAGAAGTGCT & HindIII \\
\hline HURev & CGCGTCTAGATTACGCGGCAGTTTTGC & $\mathrm{XbaI}$ \\
\hline RBPF60 & CGCGTCTAGAATTAAAGAGGAGAAATACTAGATGAACAATAAGCTGTACGTTGG & XbaI \\
\hline RBPF7 & CGCGTCTAGATCACACAGGAAACCTACTAGATGAACAATAAGCTGTACGTTGG & $\mathrm{XbaI}$ \\
\hline RBPRev & CGCGGGTACCTTAGAAGTGACCGCGACC & KpnI \\
\hline ClpPF60 & CGCGGGTACCATTAAAGAGGAGAAATACTAGATGGGACTTATACCGATGGTG & KpnI \\
\hline ClpPF7 & CGCGGGTACCTCACACAGGAAACCTACTAGATGGGACTTATACCGATGGTG & KpnI \\
\hline ClpPRev & CGCGGAATTCTCAGGTGCGCGGAC & EcoRI \\
\hline
\end{tabular}

